\title{
Cooperação entre a universidade pública e a gestão municipal no combate a COVID-19: experiências do serviço de teleatendimento em Viçosa, Minas Gerais
}

\author{
Brunnella Alcantara Chagas de Freitas ${ }^{1}$, Mara Rúbia Maciel Cardoso do Prado², Wilmara \\ Lopes Fialho ${ }^{3}$, Sophia Leonel Almeida ${ }^{4}$, Thuany Caroline Souza e Silva ${ }^{5}$, Wesley Abijaude 6
}

\begin{abstract}
Resumo: Diante do cenário da COVID-19, representantes da Universidade Federal de Viçosa e Prefeitura Municipal de Viçosa implementaram o serviço "Telessaúde Covid", fruto de um projeto de extensão, com vistas a oferecer atendimento e monitoramento por telefone aos residentes de Viçosa-MG. Até 31 de dezembro de 2020, dos 21.286 casos notificados no município, 85,5\% foram testados. Após oito meses de funcionamento, o Telessaúde realizou 3.426 novos atendimentos e 2.407 notificações, perfazendo 11,3\% do total de notificações do município, e um total de 17.071 atendimentos. O serviço tem a missão de colaborar no controle e combate a essa epidemia e de atuar no processo ativo de formação dos estudantes, aprimorando suas habilidades de comunicação e relações interprofissionais, com aquisição de competências e preparo para o mercado de trabalho. Assim, o projeto é relevante na interlocução dos envolvidos, ou seja, a sociedade, comunidade acadêmica e instituição de ensino.
\end{abstract}

Palavras-chave: Coronavírus. Telemedicina. Saúde.

Área Temática: Saúde.

\section{Cooperation between the public university and the municipal management in the fight against COVID-19: experiences of the call center service in Viçosa, Minas Gerais}

\begin{abstract}
In view of the COVID-19 scenario, representatives of the Federal University of Viçosa and the Municipality of Viçosa implemented the "Telessaúde Covid" service, the result of an extension project, with a view to offering telephone assistance and monitoring to residents of Viçosa-MG. Until December 31, 2020, of the 21,286 cases notified in the municipality, $85.5 \%$ were tested. After eight months of operation, Telehealth carried out 3,426 new calls and 2,407 notifications, making up 11.3\% of the total notifications from the municipality, and a total of 17,071 calls. The service has the mission to collaborate in the control and fight against this epidemic and to act in the active process of training students, improving their communication skills and interprofessional relationships, with the acquisition of skills and preparation for the job market. Thus, the project is relevant in the dialogue of those involved, that is, society, academic community and educational institution.
\end{abstract}

Keywords: Coronavirus. Telemedicine. Health.

\footnotetext{
${ }^{1}$ Docente do curso de Medicina, Universidade Federal de Viçosa, Departamento de Medicina e Enfermagem. E-mail: brunella.freitas@ufv.br ${ }^{2}$ Docente do curso de Enfermagem, Universidade Federal de Viçosa, Departamento de Medicina e Enfermagem. E-mail: mara.prado@ufv.br ${ }^{3}$ Docente do curso de Medicina, Universidade Federal de Viçosa, Departamento de Medicina e Enfermagem. E-mail: wilmara.fialho@ufv.br ${ }^{4}$ Discente do curso de Medicina, Universidade Federal de Viçosa, Departamento de Medicina e Enfermagem. E-mail: sophia.almeida@ufv.br ${ }^{5}$ Discente do curso de Enfermagem, Universidade Federal de Viçosa, Departamento de Medicina e Enfermagem. E-mail: thuanyvicosa@gmail.com

${ }^{6}$ Discente do curso de Enfermagem, Universidade Federal de Viçosa, Departamento de Medicina e Enfermagem. E-mail: wesley.a.abijaude@ufv.br
} 


\section{Cooperación entre la universidad pública y la dirección municipal en la lucha contra el COVID-19: experiencias del servicio de call center en Viçosa, Minas Gerais}

Resumen: Ante el escenario COVID-19, representantes de la Universidad Federal de Viçosa y el Municipio de Viçosa implementaron el servicio "Telessaúde Covid", resultado de un proyecto de extensión, para brindar atención telefónica y monitoreo a los vecinos de Viçosa-MG. Hasta el 31 de diciembre de 2020, de los 21.286 casos notificados en el municipio, se analizaron el 85,5\%. Tras ocho meses de funcionamiento, Telesalud realizó 3.426 nuevas llamadas y 2.407 notificaciones, lo que representa el 11,3\% del total de notificaciones del municipio, y un total de 17.071 llamadas. El servicio tiene la misión de colaborar en el control y lucha contra esta epidemia y actuar en el proceso activo de formación de los estudiantes, mejorando sus habilidades comunicativas y relaciones interprofesionales, con la adquisición de competencias y preparación para el mercado laboral. Así, el proyecto es relevante en la interlocución de los involucrados, es decir, sociedad, comunidad académica e institución educativa.

Palabras clave: Coronavirus. Telemedicina. Salud.

\section{INTRODUÇÃO}

Diante do cenário de saúde mundial da COVID-19, desde fevereiro de 2020, representantes da Universidade Federal de Viçosa (UFV), da Prefeitura Municipal de Viçosa (PMV), dos serviços de saúde e dos diversos setores do município passaram a se reunir e organizar para estabelecer estratégias de contingenciamento da doença na região. Paralelamente, o Ministério da Saúde, em 20 de março de 2020, publicou a Portaria n ${ }^{\circ} 467$, que dispõe, em caráter excepcional e temporário, sobre as ações de Telemedicina no âmbito do Sistema Único de Saúde (SUS), objetivando regulamentar e operacionalizar as medidas de enfrentamento da emergência de saúde pública decorrente da pandemia da COVID-19 (BRASIL, 2020).

Em vigor desde então, a portaria permite que as ações de saúde sejam realizadas durante a interação à distância, contemplando o atendimento pré-clínico, suporte assistencial, consultas, monitoramentos e diagnósticos. Esses, efetuados através de mídias digitais - como celulares, computadores e tablets —, possuem papel ímpar em conectar o profissional e o usuário, sobretudo em situações onde o contato presencial exerce alguns empecilhos à sua concretude (BRASIL, 2020; BASHSHUR et al., 2009).

Pensando nisso, uma das estratégias regionais elaborou, por meio de parceria entre o Departamento de Medicina e Enfermagem da UFV (DEM/UFV) e a Secretaria Municipal de Saúde (SMS), a implementação do serviço de telemedicina, denominado "Telessaúde Covid”. O projeto de extensão intitulado "Ações do Telessaúde Covid em Viçosa-MG: parceria entre a Universidade Federal e a Gestão Municipal” veio fomentar a integração entre os profissionais intra e extra academia, através do contato entre os cursos de Medicina e Enfermagem, Programas de Residência Médica e de Medicina veterinária da UFV, Secretaria de Saúde, Setor de Vigilância Epidemiológica e Equipe de Saúde do Município de Viçosa. Esse estímulo deu-se, além de tudo, no vislumbre de melhorias na atenção à saúde da população para o enfrentamento à COVID-19, tendo como eixo de trabalho a interface entre a integralidade, interdisciplinaridade, saúde e educação. 
Justificando o feito, a vigilância ativa e continuada dos pacientes em isolamento domiciliar mostrou-se fundamental no contingenciamento da COVID-19, sendo necessária a comunicação de um profissional de saúde com o paciente durante todo o cuidado domiciliar até o fim do período de isolamento, com revisão dos sintomas e seguimento da evolução do quadro (BRASIL, 2020; HOLLANDER; CARR, 2020; STEWART et. al., 2017). Todavia, mesmo com bons índices de resolubilidade à distância, o Telessaúde Covid preocupa-se em elaborar meios práticos de referenciar pacientes que demandam olhar presencial. Assim, quandoéidentificada a necessidade de avaliação presencial do paciente, o serviço se responsabiliza por agendar consulta na Unidade de Saúde de referência; e, quando identificada situação de gravidade, providencia transferência do paciente ao hospital destinado ao quadro.

Desse modo, no cenário da Covid-19, o serviço exerce papel fundamental ao evitar que o paciente se desloque desnecessariamente, reduzindo a sobrecarga nos serviços de saúde e a propagação do vírus na comunidade, por meio do seu adequado direcionamento, seja para acompanhamento domiciliar ou para avaliação presencial na unidade de saúde apropriada, sempre de acordo com o plano de contingência local. Ações multidimensionais e articuladas com a política de saúde local foram criadas e alinhadas junto a um sistema de vigilância existente, a fim de favorecer uma resposta satisfatória organizada e territorializada de oferta aos pacientes em relação a pandemia, fazendo com que cada paciente receba os cuidados necessários a sua queixa (BRASIL, 2020; BRASIL, 2020).

\section{OBJETIVO}

Nesse contexto, o presente artigo objetiva realizar uma análise descritiva dos resultados referentes aos oito meses de atuação do serviço de telemedicina implantada no município, em 2020.

\section{METODOLOGIA}

Trata-se de um estudo descritivo com o intuito de apresentar os resultados referentes aos oito meses de atuação do serviço de telemedicina implantada no município, no período de 22 de abril de 2020 a 31 de dezembro de 2020. O serviço compõe o projeto de extensão “Ações do Telessaúde Covid em Viçosa-MG: parceria entre a Universidade Federal e a Gestão Municipal”. Além disso, para nortear o debate, foram utilizados trinta e três documentos referenciais, nacionais e internacionais, alocados na plataforma Scielo, BVS, Pubmed e painéis de notificação regional. Para a busca, adotaram-se os descritores controlados e não controlados: Coronavírus, Telemedicina, Saúde.

\section{Apresentação do projeto}

O projeto de extensão “Ações do Telessaúde Covid em Viçosa-MG: parceria entre a Universidade Federal e a Gestão Municipal" busca oferecer atendimento e monitoramento, médico e de enfermagem, à distância 
(por telefone) a pacientes, residentes em Viçosa-MG, que entrem em contato telefônico com a equipe, por sintomas sugestivos da COVID-19 ou, por contato próximo ou domiciliar com caso confirmado ou suspeito da COVID-19, para orientações e encaminhamentos necessários, de acordo com o fluxo da rede municipal de saúde.

Quanto ao território estudado, importa dizer que Viçosa é um município brasileiro no interior do estado de Minas Gerais, Região Sudeste do país, localizada na região da Zona da Mata Mineira, com população estimada, em 2019, de 78.846 habitantes. À ótica gerencial, é sede da microrregião de saúde do seu entorno, composta por 10 municípios, ligados à Regional de Saúde de Ponte Nova. Além disso, abriga a UFV, fundada em 30 de março de 1922.

O projeto de extensão foi registrado em 22 de junho de 2020, sob o número PRJ-093/2020, embora as ações de cunho extensivo tenham se iniciado em 22 de abril de 2020. Ressaltamos que este continua em atividade no presente momento e se articula ao projeto de pesquisa "Telessaúde COVID: teleatendimento e telemonitoramento de pacientes com suspeita ou confirmação de COVID-19 no município de Viçosa-MG”, registrado no SISPPG em 03/06/2020 sob o número 40004274415 e contemplado com o Programa Institucional de Bolsas de Iniciação Científica da Universidade Federal de Viçosa 2020-2021.

\section{Público envolvido}

O público alvo do Telessaúde Covid são as pessoas residentes no município de Viçosa-MG, com sintomas sugestivos da COVID-19 ou devido a contato próximo ou domiciliar com caso confirmado ou suspeito da COVID-19, e que: (a) entram em contato com o serviço por telefone (teleatendimento), por livre demanda; (b) necessitam de monitoramento por telefone (telemonitoramento) por até 10 dias do início dos sintomas ou 14 dias do último contato, sendo provenientes do próprio teleatendimento ou das unidades de saúde do município, em seus níveis primário, secundário e terciário.

\section{Dimensionamento da equipe}

Inicialmente, entre os profissionais da rede envolvidos no serviço da SMS, estavam em ação as equipes multiprofissionais do Núcleo de Apoio à Saúde da Família (NASF) e os integrantes da Equipe Multiprofissional de Atenção Domiciliar (EMAD). O serviço ampliado passou a ser constituído por 20 estagiários bolsistas da SMS (estudantes de medicina e enfermagem da UFV), profissionais de saúde do NASF, professores/preceptores enfermeiros e médicos do DEM, e também médicos e veterinários residentes dos programas de Residência Médica e de Medicina Veterinária da UFV.

O Telessaúde Covid inicialmente funcionava por dez horas diárias, de segunda-feira a domingo e, a partir do quinto mês de funcionamento, sofreu alteração para segunda-feira a sábado e turnos de oito horas. As funções da equipe ficaram distribuídas da seguinte forma: cinco atendentes que realizam diretamente o teleatendimento e 
telemonitoramento, sendo quatro estagiários dos cursos de Medicina e Enfermagem e um profissional do NASF/ SMS; dois supervisores, um médico e um enfermeiro do DEM/UFV, que são professores, técnicos de nível superior ou médicos residentes do Programa de Residência Médica, responsáveis por supervisão dos demais profissionais em todos os turnos; um regulador, que é médico veterinário do Programa de Residência de Medicina Veterinária da UFV.

Contudo, salienta-se que houve, a partir de primeiro de novembro de 2020, a transição do serviço para gestão plena da SMS, com apoio técnico do DEM/UFV, alterando em parte o fluxo gerencial e as atribuições da equipe. O detalhamento desse processo encontra-se na seção resultados.

\section{Espaço físico e recursos tecnológicos}

O Telessaúde encontra-se em funcionamento no Laboratório de Informática do DEM/UFV e tem assessoria da Diretoria de Tecnologia da Informação. Além da existência de computadores em número suficiente, ampliaram-se os ramais, adquiriram-se headsets e extensões para o supervisor acompanhar a teleconsulta, se necessário, e foram adquiridos celular e WhatsApp para auxiliar a avaliação clínica.

O Telessaúde Covid realiza, desse modo, as consultas por telefone. Porém, outros mecanismos de contato estão sendo elaborados, como a videoconsulta, que será realizada por WhatsApp quando o atendente e supervisor julgarem necessário, e o paciente tiver recurso tecnológico para tal. Outrossim, o WhatsApp do Telessaúde Covid está apto para agilizar alguns atendimentos dificultados por ligação, realizar chamadas de vídeo ou enviar e receber documentos quando a equipe julgar necessário (como receita emitida pelo serviço, recebimento de foto de receita do paciente, entre outras situações). Por meio da função WhatsApp Business, podem-se configurar mensagens de ausência e saudação, respostas automáticas e mensagens educativas, e ofertar orientações de forma objetiva, além de separar os pacientes por categorias, como é o caso de todos os que serão monitorados de 24 em 24 horas, marcados em vermelho(BRASIL, 2020; HOLLANDER; CARR, 2020).

Criou-se, além disso, um e-mail do serviço para emissão e recebimento de documentos e para comunicação com os demais setores de saúde e com a Vigilância Epidemiológica.

\section{Treinamento e capacitação contínua da equipe para alinhamento das ações}

O treinamento inicial de toda a equipe foi realizado por videoconferência e complementado pelo apoio presencial da coordenação de professores da medicina e da enfermagem em alguns turnos no início do serviço. Priorizou-se utilizar técnicas de atendimento semelhantes ao presencial, adotando-se o Método Clínico Centrado na Pessoa (MCCP) para a entrevista clínica(GREENHALGH; KOH; CAR, 2020; STEWART et. al., 2017). Os treinamentos iniciais que visavam às medidas de prevenção da Covid-19 dentro do próprio serviço foram realizados em articulação com os profissionais do DEM/UFV, responsáveis do comitê de biossegurança da 
universidade. Através disso, mediante a entrada de novos estagiários, e pensando na fluidez de informações relacionadas a COVID-19, mantém-se a capacitação contínua da equipe com relação às atualizações emitidas pelas entidades científicas.

\section{Pactuação e articulação do fluxograma de teleatendimento e telemonitoramento com a rede de saúde local em todos os níveis de atenção}

As ações seguem o ciclo PDCA: planejar (plan), fazer (do), verificar (check) e agir (act); e, atrelado a esse arcabouço, as reuniões entre a coordenação do Telessaúde Covid e representantes da gestão de saúde ocorrem semanalmente, bem como o diálogo da coordenação com a equipe do serviço (HOLLANDER; CARR, 2020).

O Telessaúde Covid atua como um centro regulador para casos suspeitos e confirmados de Covid, onde se articulam ações com diversos setores de saúde e utilizam-se formulários semiestruturados para o teleatendimento e o telemonitoramento. (OHANNESSIAN; DUONG; ODONE, 2020). A construção, junto a isso, é reavaliada continuamente, com base em documentos do Ministério da Saúde e Secretarias Estaduais de Saúde e nos protocolos de instituições científicas, com adaptação e articulação à rede de saúde local, em todos os níveis de atenção, e de acordo com as pactuações entre a SMS e a UFV, buscando ofertar cuidado qualificado aos pacientes que buscam o amparo do serviço(BRASIL, 2020; MG, 2020; UFRGS, 2020).

\section{Divulgação do Telessaúde Covid para a população}

Aampla divulgação do serviço Telessaúde Covidé feita continuamente, nos diversos meios de comunicação, com orientações quanto às situações nas quais a população deve buscar atendimento no serviço. Além disso, há interlocução entre os setores de comunicação da PMV e da UFV para a divulgação de informações de forma coerente, sintonizando as informações nos diversos setores gerenciais e assistenciais do município.

\section{Aspectos éticos e emissão de documentos}

Em todo atendimento, é perguntado ao paciente se ele autoriza a realização do serviço por telefone. $\mathrm{O}$ atendente deve identificar adequadamente o paciente e se identificar para ele, explicando, no caso de ser estagiário, que está sendo supervisionado por médicos e enfermeiros. Todas as informações obtidas devem ser explicadas ao paciente e compartilhadas com ele, e o atendente deve checar se ficaram bem compreendidas. Os atendimentos são registrados nas folhas de atendimento e monitoramento, que são os prontuários, e, caso a equipe julgue necessário inserir informações adicionais, estas são registradas no modelo de Registro Orientado por Problemas (ReOP) (GREENHALGH; KOH; CAR, 2020; GUSSO; LOPES; DIAS, 2018).

Caso seja necessário prescrever medicação, $\mathrm{o}$ atendente deve sempre questionar o paciente sobre alergias medicamentosas, função renal e problemas de saúde prévios, além de orientar sobre possíveis alergias e efeitos 
colaterais, com registro no prontuário das perguntas e orientações (GREENHALGH; KOH; CAR, 2020; GUSSO; LOPES; DIAS, 2018).

No caso de envio de documentos necessários ao paciente, eles são encaminhados para o WhatsApp ou $e$ mail da própria pessoa, de outra instituição de saúde (acessado por médico ou enfermeiro) ou outro profissional da saúde (médico ou enfermeiro), visto que são documentos do paciente e o sigilo deve ser mantido. Esses documentos devem, prioritariamente, ter assinatura digital e estar em formato PDF, por questões de segurança (CFM, 2020).

Caso o paciente seja orientado a ficar em isolamento domiciliar, necessite de atestado para ele ou algum membro do domicílio, ou tenha indicação de Oseltamivir, ele recebe informações sobre a adoção de isolamento adequado e a possibilidade de alergia ou efeitos colaterais da medicação (BRASIL, 2020; FREITAS; PRADO; FIALHO, 2020; SBI, 2020).

O Termo de Responsabilidade de Isolamento Domiciliar e Declaração de Contatos Domiciliares deve ter sua importância e implicações devidamente explicadas pelo atendente, bem como o procedimento para o paciente dar sua anuência. O paciente é orientado sobre o procedimento adotado pela equipe para emissão de documentos (atestados ou receitas), reforçando que estes serão encaminhados após o recebimento do Termo de Responsabilidade de Isolamento Domiciliar e Declaração de Contatos Domiciliares preenchido e consentido por ele via formulário on-line (BRASIL, 2020; FREITAS, PRADO, FIALHO, 2020; UFRGS, 2020).

\section{Orçamento}

Os custos relativos aos recursos humanos foram assumidos pela SMS (profissionais de saúde e bolsas para os estagiários) e pela UFV (médicos, enfermeiros e médicos veterinários). Os custos relativos ao material permanente (computadores, aparelhos de telefone com fio, headsets) e de consumo (material impresso; equipamentos de proteção individual e para higienização: máscaras cirúrgicas, álcool a 70\%), bem como o espaço físico, foram assumidos pela UFV inicialmente. ASMS também disponibilizou, posteriormente, celular próprio do serviço para o uso do WhatsApp. Após outubro de 2020, a SMS forneceu impressora e recursos para impressão das fichas de atendimento e monitoramento e proteção e higienização (folha A4, máscaras N-95 e álcool a 70\%).

\section{Acompanhamento e avaliação}

Para a supervisão e avaliação das atividades realizadas pela equipe envolvida no projeto foram elaborados indicadores quantitativos e qualitativos. Os indicadores quantitativos foram assim definidos: (a) número das atividades realizadas; (b) carga horária de cada participante; (c) número de participantes envolvidos; (d) número de produções técnico-científicas geradas; (e) número de relatórios produzidos; (f) número de certificados expedidos; (g) indicadores de saúde da população (número de casos confirmados, descartados e curados; número de pessoas em isolamento domiciliar; número de internações; número de exames para testagem realizados, dentre outros). 
Para a avaliação dos indicadores qualitativos foram considerados: (a) elaboração de relatório bimestral e final; (b) reuniões periódicas para discussão de cada atividade desenvolvida pelo projeto visando avaliar sua influência no processo ensino-aprendizagem dos discentes; (c) percepção do modo como a relação entre a equipe do Telessaúde se estabelece com a comunidade e acerca do impacto das ações desenvolvidas pelo projeto, por meio da aplicação de questionários abertos, registro de relatos verbais em diários de campo ou realização de grupos focais.

Para a avaliação das atividades de extensão pela comunidade foram definidos indicadores qualitativos e quantitativos. Como indicadores qualitativos, definimos o modo como a relação entre o serviço e o paciente se estabelece no Telessaúde, por meio da percepção de sua adesão às orientações, manutenção do isolamento domiciliar e encaminhamentos indicados. Como indicadores quantitativos, definimos: (a) número de participantes em relação à população do município; (b) número de teleatendimentos e telemonitoramentos previstos em relação aos realizados; (c) percentuais de adesão às orientações, manutenção do isolamento domiciliar e encaminhamentos indicados; (d) impacto do Telessaúde Covid nos indicadores de saúde relacionados aos desfechos da Covid-19.

Os bolsistas e voluntários envolvidos são avaliados quanto ao compromisso, responsabilidade, dedicação, iniciativa, pontualidade e assiduidade nas atividades; conhecimentos técnicos demonstrados e capacidade de utilizá-los em situações práticas; habilidades para trabalhar com o público alvo e no preparo de materiais educativos; capacidade de sugerir modificações e soluções; organização do trabalho, cumprimento de normas e demonstração de zelo pelo patrimônio, cooperação e interação com a equipe de trabalho. Foram feitos relatórios bimestrais e um relatório final.

\section{RESULTADOS E DISCUSSÃO}

No contexto atual, o número de casos confirmados/óbitos no Brasil, em Minas Gerais e em Viçosa é de 7.675,973/194.949, 542.909/11.902 e 1.642/9, respectivamente (BRASIL, 2020). O primeiro caso confirmado de Covid-19 no município datou de 23 de abril de 2020. Até 31 de dezembro de 2020, dos 21.286 casos notificados no município, 85,5\% ( $\mathrm{n}=18.202)$ foram testados. Desses, 1.642 provaram-se casos positivos, através de confirmação laboratorial (PMV, 2020; PMV, 2020). Após oito meses de funcionamento, o Telessaúde realizou 3.426 novos atendimentos e 2.407 notificações, perfazendo 11,3\% do total de notificações do município, e um total de 17.071 atendimentos, considerando as ligações recebidas e as efetuadas para telemonitoramento.

Ainda, durante todo este período de funcionamento, a equipe constituiu-se por 27 docentes ou técnicos do DEM, 34 estudantes de graduação, 17 estudantes de pós-graduação e 4 profissionais externos.

Seguindo os princípios do atendimento presencial, os profissionais adotaram, preferencialmente, o Método Clínico Centrado na Pessoa (MCCP) para a abordagem do paciente, bem como suas queixas, demandas e 
sentimentos frente à suspeita e confirmação para a Covid-19. Na experiência do serviço, o tempo médio despendido para o primeiro atendimento foi de 30 minutos e, para o monitoramento, 15 minutos. Todavia, ressalta-se o caráter ampliado desse encontro, por vezes se adequando à necessidade do usuário.

A respeito da produção científica, um manual do serviço Telessaúde Covid, em uma primeira versão, foi construído pela equipe de coordenação, em consonância com a SMS, e com apoio da Diretoria de Comunicação Institucional da UFV na sua diagramação e divulgação on-line, no site da instituição e está disponível em: https:/ $/ w w w 2 . d t i . u f v . b r / n o t i c i a s / s c r i p t s /$ exibeNoticiaMulti.php? codNot=32586\&link=corpo. O documento visou o alinhamento das ações do Telessaúde entre a equipe e os diversos setores de saúde, desenhando, dessa forma, o fluxo de atendimento ao paciente dentro da rede de saúde local, cujas orientações poderiam ser adaptáveis a outros municípios (BRASIL, 2020; FREITAS; PRADO; FIALHO, 2020; LOEB et. al., 2020)

Considerando a necessidade de atualizações frequentes diante da pandemia da Covid-19, fez-se necessário atualizar o Manual Telessaúde Covid. Nesse sentido, foi desenvolvida a segunda edição, revista e atualizada em 14/10/2020 e disponível em: https://www2.dti.ufv.br/noticias/scripts/ exibeNoticiaMulti.php?codNot $=33330 \&$ link $=$ corpo.

Importa dizer que o serviço atravessou, posteriormente, por um processo interno de troca de gestão, a partir do dia 01 de novembro de 2020. Inicialmente a UFV entrou como gestora/coordenadora do processo de trabalho emergencialmente, com a finalidade de apoio à SMS, até que esse organizasse em termos de recursos humanos e pudesse gradativamente assumir tal função. Então, a UFV passou a integrar o apoio técnico municipal, voltando a atenção para a continuidade do projeto de pesquisa e extensão e a coordenação geral do Telessaúde Covid foi assumida pela SMS, dando sequência na prestação de serviços.

A SMS comprometeu-se quanto à manutenção dos estagiários bolsistas do DEM e designou dois enfermeiros e dois médicos, oriundos de processos contratuais da SMS, ao cargo de preceptoria dos estagiários. O DEM continuou como sede física dos atendimentos, e os recursos financeiros e materiais ficaram sob responsabilidade da Prefeitura.

Portanto, a vigilância ativa e continuada dos pacientes em isolamento domiciliar mostrou ser uma atividade fundamental para contingenciamento da Covid-19. Em razão do estreitamento da comunicação profissionalpaciente, a população tem acesso a informações adequadas e permite a continuidade do cuidado domiciliar, na maior parte das vezes, evoluindo bem e sem intercorrências até o fim do período de isolamento. E, quando identificada a necessidade de avaliação presencial, o Telessaúde Covid realiza os encaminhados necessários de acordo com a gravidade, para unidade de saúde de referência ou providencia ambulância e transferência hospitalar do paciente, de acordo com o fluxo local (BRASIL, 2020; GREENHALGH; KOH; CAR, 2020; HOLLANDER; CARR, 2020; OHANNESSIAN; DUONG; ODONE, 2020). Assim, a telemedicina não pode mais ser considerada uma opção ou complemento para o sistema de saúde reagir a uma emergência. Contudo, é necessária a continuidade de estudos para validações e superação de barreiras à sua utilização, visto a progressão da 
pandemia em vários países ao redor do globo (DORSEY; TOPOL, 2020; OHANNESSIAN; DUONG; ODONE, 2020; OMBONI, 2020).

Por conseguinte, o projeto atuou no processo ativo de formação dos estudantes, aprimorando suas habilidades de comunicação e relações interprofissionais, com interlocução de todos os envolvidos, ou seja, a sociedade, comunidade acadêmica e instituição de ensino. As vivências da extensão universitária favoreceram aos estudantes a aquisição de experiência e conhecimento e seu preparo para o mercado de trabalho.

\section{CONCLUSÕES}

A experiência de implementação do serviço Telessaúde Covid demonstrou eficiência como medida de contingenciamento da Covid-19 e é passível de replicação por outros municípios. As ações de cunho extensivo da universidade por meio do projeto de extensão "Ações do Telessaúde Covid em Viçosa-MG: parceria entre a Universidade Federal e a Gestão Municipal" destacam o número expressivo de atendimentos à população, o trabalho interdisciplinar, a atuação ativa dos estudantes no contexto, a missão social da universidade pública e a sua articulação com o sistema de saúde local.

O serviço Telessaúde Covid vem contribuindo no cenário pandêmico, devido ao grande número de atendimentos realizados desde o início, em articulação com a rede de saúde em todos os níveis, com objetivo de atender à demanda gerada pela Covid-19 e não sobrecarregar os hospitais locais. O serviço se destacou como facilitador aos pacientes no cenário atual, por levar assistência de qualidade, com segurança e seriedade nas condutas prestadas, apoio para resolver situações diversas, orientações acessíveis e eficazes e fazendo com que o paciente se sentisse seguro, acolhido e cuidado pelo serviço. Enquanto não houver ampla cobertura vacinal e a situação de emergência epidemiológica continuar, esse serviço permanecerá como facilitador e integrador das ações em saúde, com a missão de colaborar no controle e combate à epidemia.

Destarte, o projeto atuou no processo ativo de formação dos estudantes, aprimorando suas habilidades de comunicação e relações interprofissionais, com aquisição de competências e preparação para o mercado de trabalho. Assim, identificou-se a relevância do projeto na interlocução dos envolvidos, ou seja, a sociedade, comunidade acadêmica e instituição de ensino.

\section{AGRADECIMENTOS}

Os autores do trabalho agradecem ao Programa institucional de bolsas de iniciação científica da Universidade Federal de Viçosa - PIBIC/UFV e à Secretaria Municipal de Saúde (SMS) da Prefeitura de Viçosa (PMV) pela parceria e apoio financeiro; à Universidade Federal de Viçosa (UFV) e ao Departamento de Medicina e Enfermagem (DEM/UFV) pela oferta e manutenção do espaço físico, recursos materiais e humanos durante as atividades realizadas; à Divisão de saúde da UFV por fornecer materiais para o processo de desinfecção do espaço físico; aos Programas de Residência Médica e de Residência em Medicina Veterinária da UFV pela 
participação com recursos humanos; À DTI da UFV, que ampliou as linhas telefônicas; e a todos os estagiários e discentes envolvidos no atendimento, triagem e monitoramento, sobretudo pela atenção, disponibilidade, resolubilidade e humanidade ao se encontrarem com os pacientes.

\section{FONTES DE FINANCIAMENTO}

Durante o percurso, o projeto recebeu auxílio financeiro do Programa institucional de bolsas de iniciação científica da Universidade Federal de Viçosa - PIBIC/UFV, Edital de seleção 2020-2021, além dos recursos destinados pela Secretaria Municipal de Saúde, para bolsas de estagiários dos cursos de graduação de enfermagem e medicina.

\section{REFERÊNCIAS}

BACHSHUR, Rashid L.; SHANNON, Gary W.; KRUPINSKI, Elizabeth A.; GRIGSBY, Jim; KVEDAR, Joseph C.; WEINSTEIN, Ronald S.; SANDERS, Jay H.; RHEUBAN, Karen S.; NESBITT, Thomas S.; ALVERSON, Dale C.; MERRELL, Ronald C.; LINKOUS, Jonathan D.; FERGUSON, A. Stewart; WATERS, Robert J.; STACHURA, Max E.; ELLIS, David G.; ANTONIOTTI, Nina M.; JOHNSTON, Barbara; DOAM, Charles R.; YELLOWLESS, Peter; NORMANDIN, Steven; TRACY, Joseph. National Telemedicine Initiatives: essential to healthcare reform. Telemedicine And E-Health, v. 15, n. 6, p. 600-610, 2009. Disponível em: <https://pubmed.ncbi.nlm.nih.gov/19534591/>. Acesso em: 2 Jun. 2020.

BRASIL. Ministério da Saúde. Portaria n ${ }^{\circ} 467$, de 20 de março de 2020. Dispõe, em caráter excepcional e temporário, sobre as ações de Telemedicina, com o objetivo de regulamentar e operacionalizar as medidas de enfrentamento da emergência de saúde pública de importância internacional previstas no art. $3^{\circ}$ da Lei ${ }^{\circ}$ 13.979, de 6 de fevereiro de 2020, decorrente da pandemia de COVID-19. Brasília, 2020. Disponível em: <http://www.planalto.gov.br/CCIVIL_03/Portaria/PRT/Portaria\%20n\%C2\%BA\%20467-20ms.htm>. Acesso: 7 Mai. 2020.

BRASIL. Ministério da Saúde. Secretaria de Atenção Primária à Saúde. Protocolo de manejo clínico do coronavírus (Covid-19) na atenção primária à saúde. Brasília, 2020. Disponível em: <https:// www.unasus.gov.br/especial/covid19/pdf/37>. Acesso: 7 Mai. 2020.

BRASIL. Ministério da saúde. Secretaria de Vigilância em Saúde. Boletim epidemiológico coronavírus. 2021. Disponível em: <https://www.gov.br/governodigital/pt-br/acessibilidade-digital>. Acesso em: 24 Mar. 2021.

BRASIL. Ministério da Saúde. Secretaria de Vigilância em Saúde. Departamento de Imunização e Doenças Transmissíveis. Coordenação-Geral do Programa Nacional de Imunizações. Parecer técnico $n^{\circ}$ 67/2020CGPNI/DEIDT/SVS/MS. Indicação, em caráter excepcional, do uso do fosfato de Oseltamivir durante a pandemia da Covid-19. Brasília, 2020. 5 p. Disponível em: <https://www.cosemssc.org.br/parecer-tecnico-no67-2020-cgpni-deidt-svs-ms/>. Acesso em: 18 Mai. 2020.

Centers for Disease Control and Prevention - CDC. Interim clinical guidance for management of patients with confirmed coronavirus disease (Covid-19). Georgia, 2020. Disponível em: <https://www.cdc.gov/ coronavirus/2019-ncov/hcp/clinical-guidance-management-patients.html>. Acesso em: 28 Jun. 2020. 
COLE, Steven.; BIRD, Julian. The Medical Interview: The Three Function Approach. 3. ed. Filadélfia: Saunders Elsevier, 2013.336 p.

Conselho Federal de Medicina - CFM. Entra em funcionamento serviço que permite validar receitas médicas e atestados digitais. Brasília, 2020. Disponível em: <http://portal.cfm.org.br/ index.php?option=com_content\&view=article\&id=28674:2020-04-23-13-38-34\&catid=3\#: :text=O Conselho Federal de Medicina,Telemedicina\%2C emitir atestados ou receitas>. Acesso em: $30 \mathrm{Abr} .2020$.

DORSEY, Earl Ray; TOPOL, Eric Jeffrey. Telemedicine 2020 and the next decade. The Lancet, v. 395, n. 10227, p. 859, 2020. Disponível em: <https://www.thelancet.com/journals/lancet/article/piis01406736(20)30424-4/fulltext\#\%20>. Acesso em: 2 Jun. 2020.

Governo do Estado do Espírito Santo. Secretaria de Saúde. Nota Técnica COVID-19 n 03/2020. Definições de afastamento laboral para profissionais de serviços essenciais. Espírito Santo, 2020. 3 p. Disponível em: <https://saude.es.gov.br/media/sesa/coronavirus/notas\%20t\%c3\%a9cnicas/ nota\%20t\%c3\%89cnica\%20covid.19\%20n.\%2003.20.\%20defini\%c3\%a7\%c3\%b5es\%20de\%20 afastamento\%20laboral.pdf> . Acesso em: 28 Jun. 2020.

FREITAS, Brunnella Alcantara Chagas de; PRADO, Mara Rubia Maciel Cardoso do; FIALHO, Wilmara Lopes. Telessaúde Covid: teleatendimento e telemonitoramento de pacientes com suspeita ou confirmação de Covid-19. Parceria entre o Departamento de Medicina e Enfermagem da Universidade Federal de Viçosa e a Secretaria Municipal de Saúde de Viçosa-MG. Viçosa, 2020. E-book. 65 p. Disponível em: <https://www2.dti.ufv.br/noticias/files/anexos/1591023147.pdf〉. Acesso em: 2 Jun 2020.

Governo do Estado de Minas Gerais, Belo Horizonte. Secretaria de Estado de Saúde, Centro de Operações de Emergência em Saúde. Atualização técnica ao protocolo de infecção humana pelo Sars-Cov- $2 n^{\circ} 02 /$ 2020 - 23/03/2020. Definições de casos operacionais e fluxos de testagem laboratorial e notificação dos casos. Belo Horizonte, 2020. Disponível em: <https://www.saude.mg.gov.br/images/noticias_e_eventos/ 000_2020/24-03-Atualizacao_Protocolo-02-Casos-Operacionais-e-Fluxos-de-Testagem-LaboratorialNotificacao-dos-Casos.pdf>. Acesso em: 3 abr. 2020.

Governo do Estado de Minas Gerais, Belo Horizonte. Secretaria de Estado de Saúde, Centro de Operações de Emergência em Saúde. Atualização técnica ao protocolo de infecção humana pelo Sars-Cov-2 $n^{\circ} 05 /$ 2020 - 19/05/2020. Definições de casos operacionais e fluxos de testagem laboratorial e notificação dos casos. Belo Horizonte, 2020. Disponível em: <https://www.itauna.mg.gov.br/arquivos/19-05-atualizacaoprotocolo_20115027.pdf>. Acesso em: 28 jun 2020.

Governo do Estado de Minas Gerais, Belo Horizonte. Secretaria de Estado de Saúde, Centro de Operações de Emergência em Saúde. Nota técnica Coes Minas Covid-19 no 8 - 20/03/2020. Orientações sobre ações frente ao enfrentamento da infecção humana pelo Sars-Cov-2 (doença pelo coronavírus - Covid-19) para as equipes do Programa Melhor em Casa - Serviço de Atenção Domiciliar. Belo Horizonte, 2020. Disponível em: <https://www.saude.mg.gov.br/coronavirus/profissionaisdesaude>. Acesso em: 20 abr. 2020.

GREENHALGH, Trisha; KOH, Gerald Choon Huat; CAR, Josip. COVID-19: a Remote Assessment in Primary Care. BMJ, 368 :m1182, 2020. DOI:10.1136/bmj.m1182. Disponível em: <https://www.bmj.com/ content/368/bmj.m1182.abstract>. Acesso em: 7 mai. 2020.

GUSSO, Gustavo; LOPES, José Mauro Ceratti; DIAS, Chaves Lêda. Tratado de Medicina de Família e Comunidade: princípios, formação e prática. 2a ed. Porto Alegre: Artmed, 2018. 
HOLLANDER, Judd; CARR, Brendan. Virtually perfect? Telemedicine for COVID-19. New England Journal of Medicine, 2020. Disponível em: <https://www.nejm.org/doi/full/10.1056/nejmp2003539>. Acesso em: 7 abr. 2020.

LOEB, Alexander E.; RAO, Sandesh S.; FICKE, James R.; MORRIS, Carol D.; RILEY, Lee H.; LEVIN, Adam S. Departmental Experience and Lessons Learned With Accelerated Introduction of Telemedicine During the COVID-19 Crisis. Journal of the American Academy of Orthopaedic Surgeons, v. 11, n. 28, p. 469-476, 2020. Disponível em: <https://pubmed.ncbi.nlm.nih.gov/32301818>. Acesso em: 20 jun. 2020.

OHANNESSIAN, Robin; DUONG, Tu Anh; ODONE, Anna. Global Telemedicine Implementation and Integration Within Health Systems to Fight the COVID-19 Pandemic: a A Call to Action. JMIR Public Health Surveill, v. 6, n. 2, 2020. Disponível em: <https://publichealth.jmir.org/2020/2/e18810>. Acesso em: 20 jun. 2020.

OMBONI, Stefano. Telemedicine During the Covid-19 in Italy: A Missed Opportunity? Telemedicine and eHealth, v. 26, n. 8, p. 973-975, 2020. Disponível em: <https://www.ncbi.nlm.nih.gov/pmc/articles/ PMC7415870/>. Acesso em: 20 jun. 2020.

Prefeitura Municpal de Viçosa, Secretaria Municipal de Saúde. Boletim Epidemiológico n.43. 2020. Disponível em: <https://www.vicosa.mg.gov.br/abrir_arquivo.aspx/ Boletim_Coronavirus_43?cdLocal $=2 \&$ arquivo $=\{$ 5DED6EA2-DB10-AB25-AEAC2B7D73CC3ED4\}.pdf>. Acesso em: 8 mar. 2021.

Prefeitura Municpal de Viçosa, Secretaria Municipal de Saúde. Boletim Epidemiológico n.295. 2020. Disponível em: <https://www.vicosa.mg.gov.br/abrir_arquivo.aspx/ 31_12_2020_Boletim_Coronavirus_295?cdLocal=2\&arquivo $=\{$ E6CB3A1E-D26B-66EC-2BB8B116D7CCC348 \}.pdf>. Acesso em: 8 marc. 2021.

Sociedade Brasileira de Infectologia - SBI. Orientações sobre diagnóstico, tratamento e isolamento de pacientes com Covid-19. 2020. Disponível em: <https://infectologia.org.br/>. Acesso em: 3 mai. 2020.

STEWART, Moira; BROWN, Judith Belle; WESTON, W. Wayne; MCWHINNEY, Ian R.; MCWILLIAM, Carol L.; FREEMAN, Thomas R. Medicina Centrada na Pessoa: transformando o método clínico. 3a ed. Porto Alegre: Artmed. 2017.

Universidade Federal do Rio Grande do Sul - UFRGS, Telessaúde Rio Grande do Sul. Orientações para os casos com indicação de isolamento domiciliar. 2020. Disponível em: <https://www.ufrgs.br/telessauders/documentos/ telecondutas/material_isolamento_corona_virus_20200303_ipn_002.pdf>.Acesso em: 15 mai. 2020.

Universidade Federal do Rio Grande do Sul - UFRGS. Coronavírus (Covid-19): informações para profissionais da APS. Porto Alegre: Faculdade de Medicina. 2020. Disponível em: <https://www.ufrgs.br/ telessauders/teleconsultoria/0800-644-6543/\#telecondutas-0800>. Acesso em: 7 mai. 2020.

Submetido em: 19/04/2021 Aceito em: 07/06/2021 